\title{
ABSOLUTE INTEGRAL CLOSURES ARE BIG COHEN-MACAULAY ALGEBRAS IN CHARACTERISTIC P
}

\author{
MELVIN HOCHSTER AND CRAIG HUNEKE
}

Throughout this paper "ring" means commutative ring with identity and modules are unital. Our main interest is in local rings, i.e., Noetherian rings $(R, m)$ with a unique maximal ideal $m$. In such a ring, $x_{1}, \ldots, x_{n} \in m$ is a system of parameters if $m$ has a power in the ideal $\left(x_{1}, \ldots, x_{n}\right) R$ and $n$ is the Krull dimension of $R$. When $R$ is complete and contains a field, this means that $R$ is module-finite over a formal power series subring $K\left[\left[x_{1}, \ldots, x_{n}\right]\right]=A . \quad R$ is called Cohen-Macaulay if some (equivalently, every) system of parameters is a regular sequence in $R$, which means that every $x_{i+1}$ is a nonzerodivisor on $R /\left(x_{1}, \ldots, x_{i}\right) R$, for $0 \leq i \leq n-1$. In the case where $R$ is module-finite over the formal power series subring $A$, this means that $R$ is a free $A$-module. For many theorems of commutative algebra and of algebraic geometry, the Cohen-Macaulay condition (possibly on the local rings of a variety) is just what is needed to make the theory work. Our main result, which is given below in an algebraic form in Theorem 1 and in a geometric form in Theorem 2, asserts that, under mild conditions on a local ring $R$ of positive prime characteristic $p$, one can "correct" the failure of the Cohen-Macaulay condition in $R$ itself by passing to a very large integral extension of $R$. It is worth emphasizing that this is quite false in characteristic 0 , and comes as a surprise, we believe, in characteristic $p$.

One can get an idea of how far these theorems are from the truth over a field of characteristic 0 from the following observation: In the situation of Theorem 2 , when $X$ is projectively normal, the maps of cohomology are always injective, by an easy trace argument. Despite the fact that both Theorem 1 and Theorem 2

Received by the editors February 12, 1990 and, in revised form, May 24, 1990. 1980 Mathematics Subject Classification (1985 Revision). Primary 13B20, 13H99, $13 \mathrm{C} 99$.

Both authors were supported in part by the National Science Foundation. 
are false in equal characteristic 0 , they have deep consequences in that case.

We denote by $R^{+}$the integral closure of the domain $R$ in an algebraic closure of its fraction field (the absolute integral closure in the sense of Artin [Ar]). Let $\mathbf{N}$ be the nonnegative integers. If $R$ is a finitely generated $\mathrm{N}$-graded domain over $R_{0}=K$, a field, there is a maximum subring $R^{+g r}$ of $R^{+}$to which the $\mathrm{N}$-grading extends. Our main result, which is proved in detail in [HH8], is:

Theorem 1. If $R$ is an excellent local domain (respectively, a finitely generated $\mathbf{N}$-graded domain over a field) of characteristic $p$, then every system of parameters in the Jacobson radical of $R$ (respectively, every homogeneous system of parameters) is a regular sequence on $R^{+}$(respectively, on $R^{+g r}$ ). In consequence, every equicharacteristic local ring $R$ can be mapped by a local homomorphism to a quasilocal ring $S$ such that every system of parameters for $R$ maps to a regular sequence in $S$.

Note that every local ring essentially of finite type over a field and every complete local ring is excellent.

An easy corollary of the graded version of Theorem 1 is:

Theorem 2. Let $X$ denote a closed subscheme of projective $n$-space over a field $K$ of characteristic $p$. Suppose that $X$ is reduced and that all irreducible components have the same dimension, say, $d$. Then for all $i, 1 \leq i<d$, and for every integer $t$ there is a finite surjective morphism $f_{i t}=f: Y \rightarrow X$ from a projective scheme $Y$ over a finite algebraic extension $K^{\prime}$ of $K$ to $X$, such that the induced map $H^{i}\left(X, \mathscr{O}_{X}(t)\right) \rightarrow H^{i}\left(Y, f^{*} \mathscr{O}_{X}(t)\right)$ is $0 . \quad Y$ may be chosen to be reduced and such that all irreducible components have dimension $d$. If $X$ is reduced and irreducible, then $Y$ may be chosen reduced and irreducible.

In particular, if $K$ is a field of characteristic $p$ and $S$ denotes the integral closure of the power series ring $K\left[\left[x_{1}, \ldots, x_{n}\right]\right]$ in an algebraic closure of its fraction field, then $x_{1}, \ldots, x_{n}$ is a regular sequence in $S$. Even better, as a consequence of the same ideas, $S$ is flat over $K\left[\left[x_{1}, \ldots, x_{n}\right]\right]$. In the graded case, one has that if $R=K\left[x_{1}, \ldots, x_{n}\right]$ is a polynomial ring and $S=R^{+g r}$ then $S$ is a free $R$-module.

Many corollaries ensue. One example is the existence of big Cohen-Macaulay modules in equicharacteristic (cf. [Ho2]) but 
now one gets a stronger result: One has big Cohen-Macaulay algebras. It also follows that if a locally excellent domain $R$ of characteristic $p$ splits from all module-finite extensions, then $R$ is Cohen-Macaulay. From this result one can deduce the characteristic $p$ case of the theorem that a direct summand of an equicharacteristic regular ring is Cohen-Macaulay. By a different line of argument, which is sketched in the next paragraph, one can deduce the equicharacteristic 0 case as well, and this yields the theorem that a ring of invariants of a reductive group acting on a regular ring of characteristic 0 has a Cohen-Macaulay ring of invariants (cf. [HR, Bou]).

One proves first that if $R$ is an equicharacteristic module-finite torsion-free extension of the regular Noetherian domain $A$ and $S$ is any regular ring that is an $R$-algebra, then for every finitely generated $A$-module $M$, the map $\operatorname{Tor}_{i}^{A}(M, R) \rightarrow \operatorname{Tor}_{i}^{A}(M, S)$ vanishes for all $i \geq 1$. (A weaker version of this result can be obtained by tight closure methods: see [HH4].) One reduces to the case where all the rings are complete local, $R$ is a domain, and the map $R \rightarrow S$ is onto. One can use Artin approximation to reduce to the case of affine algebras, and then pass to characteristic $p$ by standard techniques. The key point in characteristic $p$ is that one gets a map $R^{+} \rightarrow S^{+}$, and $R^{+}$may be identified with $A^{+}$and thus is $A$-flat. The composite map $\operatorname{Tor}_{i}^{A}(M, R) \rightarrow \operatorname{Tor}_{i}^{A}(M, S) \rightarrow \operatorname{Tor}_{i}^{A}\left(M, S^{+}\right)$then vanishes for $i \geq 1$ because it also factors through $\operatorname{Tor}_{i}^{A}\left(M, R^{+}\right)=0$, but the second arrow is injective because, since $S$ is regular, it is pure in $S^{+}$(cf. [Ho1]). One can apply this in the case where $R \rightarrow S$ splits to prove that $R$ is Cohen-Macaulay. One first reduces to the case where $R$ is complete local, so that $R$ is module-finite over a regular ring $A$. The fact that $R \rightarrow S$ splits coupled with the result just proved then implies that $\operatorname{Tor}_{i}^{A}(M, R)=0$ for all $i \geq 1$ and all finitely generated $R$-modules $M$. This in turn yields that $R$ is $A$-flat and, hence, Cohen-Macaulay.

This argument illustrates a general principle in the application of both Theorem 1 and Theorem 2. In both cases one gets maps that kill cohomology. But one often has extra hypotheses that make the maps injective, and so the cohomology vanishes. Both theorems give the intuition that in many situations, both geometric and algebraic, one can expect behavior that is more like the Cohen-Macaulay case than would have seemed plausible before these theorems were proved. 
The reader is referred to [HH8, HH9] for details. Theorem 1 also yields a new proof in characteristic $p$ (and, by standard techniques, then also in characteristic 0 ) of a connectedness theorem of Faltings [Fa1], which asserts that the punctured spectrum of a closed subscheme of $\operatorname{Spec} R$, where $R$ is a complete local domain of dimension $d$, defined by the vanishing of at most $d-2$ elements, is connected. One can reduce to the case where the elements are part of a system of parameters. By passing to $R^{+}$one can give, in essence, the same proof as for the case of a CohenMacaulay ring, which is easy. See [HH8, §6].

We also observe the following seemingly simple but apparently new corollary of our results in the graded case: Consider an $\mathbf{N}$ graded affine algebra over an algebraically closed field and let $F_{1}$, $\ldots, F_{k}$ be homogeneous parameters of degree $m$. Then any relation on the $F_{i}$ with coefficients of degree $m$ is trivial. This result can be applied to problems in enumerative geometry. See [HH9].

We note that the results presented here grew out of techniques developed to study tight closure (cf. [HH1-HH7]).

The rest of our remarks are devoted to sketching the proof of Theorem 1 (for simplicity, we do not keep track of the graded case). We define some notation, indicate several lemmas, and then sketch the argument. If $I \subseteq R$ where $R$ is a ring of characteristic $p$ and $q=p^{e}$, we denote by $I^{[q]}$ the ideal generated by the $q$ th powers of the elements of $I$. An embryonic version of Theorem 3 below, which is our main tool for forcing elements into expansions of ideals in module-finite extension rings and so is crucial to the proof of Theorem 1, is given in [Ma] for the case where $k=2$ and $I$ is principal. That case is much easier, but it, along with the results of [HH6], suggested that the right form for:

Theorem 3. If $R$ is a ring of characteristic $p, I$ is an ideal generated by $x_{1}, \ldots, x_{k}$, each of which is a nonzerodivisor in $R$, and $J$ is a finitely generated ideal of $R$ such that $J^{[p]} \subseteq I^{[p]}+$ $\left(x_{1} \cdots x_{k}\right)^{p-1} J$, then $J \subseteq I S \cap R$ for some module-finite overring $S$ of $R$. Moreover, $S$ can be taken to be a domain if $R$ is a domain.

If $R$ is an excellent semilocal ring with Jacobson radical $J$ in which (0) has no embedded prime and such that for every minimal prime $P$ and maximal ideal $m \supseteq P, \operatorname{dim} R_{m} / P R_{m}=$ $\operatorname{dim} R$, we shall say that $R, J$ satisfy the standard hypotheses. If $R, J$ satisfy the standard hypotheses and $z \in R$, we shall say that 
$z$ is a colon-killer (respectively, a weak colon-killer) if for every sequence $x_{1}, \ldots, x_{k}$ of elements of $J$ that is part of a system of parameters (we refer to these as a sequence of parameters) we have $z\left(\left(x_{1}, \ldots, x_{k-1}\right) R:_{R} x_{k} R\right) \subseteq\left(x_{1}, \ldots, x_{k-1}\right) R$ (respectively, $z^{t}\left(\left(x_{1}, \ldots, x_{k-1}\right) R:_{R} x_{k} R\right) \subseteq\left(x_{1}, \ldots, x_{k-1}\right) R$ for some positive integer $t$, which may depend on what the sequence $x_{1}, \ldots, x_{k}$ is).

Note that in the above definition we could have used arbitrary positive integer powers $x_{i}^{a_{i}}$ instead of the original $x_{i}$, since replacing the $x$ 's by powers simply produces a new sequence of parameters.

Lemma 4. If $R$ satisfies the standard hypotheses and $x$ is an element such that $R_{x}$ is a Cohen-Macaulay ring, then $x$ has a power which is a colon-killer. (See [Ro] or [HH3, §11].)

If $I$ is an ideal of a Noetherian ring $R$, we denote by $I^{u}$ the intersection of the primary components of $I$ corresponding to minimal primes of $I$. We say that $x, x_{1}, \ldots, x_{k} \in J$ constitute an extraordinary sequence of parameters if they are elements that are part of a system of parameters such that (1) $x$ is a weak colon-killer and (2) for $1 \leq i<k$, the image of $x_{i}$ in the ring $R /\left(\left(x_{1}, \ldots, x_{i-1}\right) R\right)^{u}$ is a colon-killer. If $R$ is a ring, $I \subseteq R$ is an ideal, and $x \in R$, then we let $I:_{R} x^{\infty}=\bigcup_{t} I:_{R} x^{t} R$, the contraction of $I R_{x}$ to $I$.

Theorem 5. If $R, J$ satisfy the standard hypotheses, $x, x_{1}, \ldots, x_{k}$ is an extraordinary sequence of parameters, and $a_{1}, \ldots, a_{k}$ are arbitrary positive integers, then

$$
\begin{gathered}
\left(x_{1}^{a_{1}}, \ldots, x_{k}^{a_{k}}\right) R:_{R} x^{\infty} \\
=\sum_{i=0}^{k-1}\left(\prod_{\nu=1}^{i} x_{\nu}^{a_{\nu}-1}\right)\left(\left(x_{1}, \ldots, x_{i}, x_{i+2}^{a_{i+2}}, \ldots, x_{k}^{a_{k}}\right) R:_{R} x^{\infty}\right) \\
\quad+\left(\prod_{\nu=1}^{k} x_{\nu}^{a_{\nu}-1}\right)\left(\left(x_{1}, \ldots, x_{k}\right) R:_{R} x^{\infty}\right) .
\end{gathered}
$$

Lemma 6. Let $R, J$ satisfy the standard hypotheses, where $R$ is of positive prime characteristic $p$, and let $x, x_{1}, \ldots, x_{k}$ be an extraordinary sequence of parameters. Let $I=\left(x_{1}, \ldots, x_{k}\right) R:_{R} x^{\infty}$. Suppose that every proper subset of $x_{1}, \ldots, x_{k}, x$ forms a regular sequence in $S=R^{+}$. Then $I \subseteq\left(x_{1}, \ldots, x_{k}\right) S$. 
Proof. $I^{[p]} S \subseteq\left(x_{1}^{p}, \ldots, x_{k}^{p}\right) S+\left(x_{1}, \ldots, x_{k}\right)^{p-1} I S$, by Theorem 5 , and then Theorem 3 yields the result.

Suppose that $R, J$ satisfy the standard hypotheses. We say that a sequence of parameters $y_{0}, \ldots, y_{k}$ in $J$ is derived from a sequence of parameters $x_{0}, \ldots, x_{k}$ if it can be obtained from $x_{0}, \ldots, x_{k}$ by a finite sequence of operations of three types: (1) permuting the $x_{i} ;(2)$ replacing $x_{i}$ by $x_{i}+r x_{j}$ for $j \neq i, r \in R$; and (3) replacing $x_{i}$ by $y x_{i}$, where $y \in J$ is any element such that $x_{0}, \ldots, x_{i-1}, y, x_{i+1}, \ldots, x_{k}$ is a sequence of parameters (in particular, one may replace $x_{i}$ by $x_{i}^{t}$, where $t$ is a positive integer).

Lemma 7. Let $R, J$ satisfy the standard hypotheses and let $\mathbf{x}=$ $x_{0}, \ldots, x_{k}$ be a sequence of parameters in $J$. Let $S=R^{+}$. Suppose that $\mathbf{y}=y_{0}, \ldots, y_{k}$ is a sequence of parameters in $J$ derived from $\mathbf{x}$. Finally, suppose that every sequence of parameters of length $k$ in $R$ forms a regular sequence in $S$. Then if all the relations in $R$ on the $y_{i}$ become trivial in $S$, the same is true for the $x_{j}$.

Lemma 8 (Moving Lemma). Let $R, J$ satisfy the standard hypotheses and let $x_{0}, \ldots, x_{k}$ be any sequence of parameters in $J$. Then there is a derived sequence $y_{0}, \ldots, y_{k}$ which is an extraordinary sequence of parameters.

Sketch of the proof of Theorem 1. Use induction on the number $k$ of parameters in the sequence. Lemmas 8 and 7 permit one to assume the sequence is extraordinary, and Lemma 6 allows one to reduce to the problem of killing a relation on $k-1$ elements.

\section{REFERENCES}

[Ar] M. Artin, On the joins of Hensel rings, Adv. Math. 7 (1971), 282-296.

[Bou] J.-F. Boutot, Singularités rationelles et quotients par les groupes réductifs, Invent. Math. 88 (1987), 65-68.

[Fal] G. Faltings, A contribution to the theory of formal meromorphic functions, Nagoya Math. J. 77 (1980), 99-106.

[Ho1] M. Hochster, Contracted ideals from integral extensions of regular rings, Nagoya Math. J. 51 (1973), 25-43.

[Ho2] _ Topics in the homological theory of modules over commutative rings, CBMS Regional Conf. Ser. in Math., no. 24, Amer. Math. Soc., Providence, R.I., 1975.

[HH1] M. Hochster and C. Huneke, Tight closure, Commutative Algebra, M.S.R. I. Publ., no. 15, Springer-Verlag, New York, 1989, pp. 305-324. 
[HH2] —, Tight closure and strong F-regularity, Mém. Soc. Math. de France numéro consacré au colloque en l'honneur de P. Samuel, Mémoire $n^{\circ} 38$ (1989), 119-133.

[HH3] _. Tight closure, invariant theory, and the Briançon-Skoda theorem, J. Amer. Math. Soc. 3 (1990), 31-116.

[HH4] _ Phantom homology, preprint.

[HH5] _- F-regularity, test elements, and smooth base change (in preparation).

[HH6] _ - Tight closures of parameter ideals and splitting in module-finite extensions (in preparation).

[HH7] _ - Tight closure in characteristic zero (in preparation).

[HH8] _ Infinite integral extensions and big Cohen-Macaulay algebras, preprint.

[HH9] _ Applications of the Cohen-Macaulay property for absolute integral closures (in preparation).

[HR] M. Hochster and J. L. Roberts, Rings of invariants of reductive groups acting on regular rings are Cohen-Macaulay, Adv. Math. 13 (1974), 115-175.

[Ma] F. Ma, Splitting in integral extensions, Cohen-Macaulay modules and algebras, J. Algebra 116 (1988) 176-195.

[Ro] P. Roberts, Two applications of dualizing complexes over local rings, Ann. Sci. École Norm. Sup. 9 (1976), 103-106.

Mathematics Department, University of Michigan, ANn ARbor, Michigan 48109-1003

Mathematics Department, Purdue University, West Lafayette, INDIANA 47907 
